Johannes Mayr

\title{
Inflationsangst in Deutschland - Vorsicht vor zu engem Blick auf Konjunktur und Geldmenge
}

Die Inflationsrate in Deutschland ist im Zuge der Erholungsbewegung nach der Corona-Krise wie erwartet deutlich gestiegen, auf zuletzt 3,9\% im August 2021. Die Bundesbank hält einen Anstieg bis auf $5 \%$ in den kommenden Monaten für möglich (Bundesbank, 2021a). In den anderen Euroländern und den USA ist die Entwicklung ähnlich. Die Mehrheit der Ökonom:innen wie auch die Notenbanken gehen aber davon aus, dass die Inflationsraten im Verlauf des Jahres 2022 wieder in den Bereich der Zielwerte der Geldpolitik zurückfallen werden, oder sogar darunter. Die Argumente für einen nur temporären Charakter des Inflationsanstiegs liegen auf der Hand. So werden die Preise aktuell stark durch Sondereffekte infolge der Corona-Krise, der Wiedereröffnungen sowie der wirtschaftspolitischen Maßnahmen nach oben gedrückt. In Deutschland zählt neben der temporären Mehrwertsteuersenkung im zweiten Halbjahr 2020 die Ausweitung der $\mathrm{CO}_{2}$-Bepreisung zum Jahresanfang 2021 zu den zentralen Treibern (Destatis, 2021). Dazu kommen Preisanstiege durch Knappheiten bei Rohstoffen und Vorprodukten. Für diese Engpässe sorgt vor allem ein schleppendes Tempo beim Wiederhochfahren der Produktion, während die Nachfrage im ersten Halbjahr 2021 in vielen Bereichen rasch wieder angesprungen ist.

Die Notenbanken bauen darauf, dass diese Sondereffekte in den kommenden Monaten an Bedeutung verlieren. Auch die Investierenden halten diese Erwartungen für realistisch. An den Finanzmärkten wird weiterhin lediglich ein Inflationsbuckel gepreist, für die USA wie auch für den Euroraum und Deutschland. Gleichzeitig werden die Aufwärtsrisiken für die Preisstabilität vor allem in Deutschland von Ökonom:innen und der Öffentlichkeit hitzig diskutiert, meist vom Standpunkt eines zu expansiven Kurses der Geld- und/oder Fiskalpolitik aus. Häufig schwingt

(C) Der/die Autor:in 2021. Open Access: Dieser Artikel wird unter der Creative Commons Namensnennung 4.0 International Lizenz veröffentlicht (creativecommons.org/licenses/by/4.0/deed.de).

Open Access wird durch die ZBW - Leibniz-Informationszentrum Wirtschaft gefördert.

Dr. Johannnes Mayr ist Chefvolkswirt bei der Eyb \& Wallwitz Vermögensmanagement $\mathrm{GmbH}$ in München. dabei der Verdacht von Zugriffen des Staates oder der Partnerländer der Währungsunion auf die Vermögen und Ersparnisse durch die Hintertür mit. Regelmäßig werden die historischen Erfahrungen Deutschlands mit der Hyperinflation in den 1920er Jahren angeführt. Zielführend ist eine solche Orientierung kaum. Zum einen sind die Treiber der Inflation in den 1920er Jahren komplexer als häufig suggeriert. Zum anderen ist die Vergleichbarkeit der Perioden durch die stark veränderten gesellschaftlichen, wirtschaftlichen und politischen Rahmenbedingungen begrenzt. Problematisch ist dieser Vergleich auch deshalb, da strukturelle Treiber von Preisrisiken dadurch in den Hintergrund gedrängt werden.

\section{Konjunktur schäumt nicht über und Geldmenge bleibt passiv}

Aktuell werden Inflationsrisiken sowohl auf Angebots- als auch auf Nachfrageseite diskutiert. Auf der Angebotsseite geht der Blick in Richtung höhere Rohstoffpreise und Engpässe bei Vorprodukten durch Produktionsstörungen sowie politische und regulatorische Maßnahmen (z.B. Steuer- und Zollanhebungen), die auf die Verkaufspreise überwälzt werden. Bei der Nachfrage wird befürchtet, dass ein Rückgang der Sparneigung, das geringe Zinsniveau und der Anstieg der Geldmenge sowie eine zu expansive Fiskalpolitik die Nachfrage über das wenig flexible Angebot hinaustreiben und so einen steigenden Preisdruck auslösen könnten. Gemäß der gängigen Prognosemodelle auf Basis der Neu-Keynesianischen Phillipskurve sorgt in beiden Fällen vor allem der mit den Primäreffekten verbundene Anstieg der Inflationserwartungen für eine veränderte Preissetzung der Unternehmen und eine höhere Zahlungsbereitschaft der Haushalte und treibt die tatsächliche Inflation nach oben. Während die aktuelle Inflationsdynamik und die kurzfristigen Aufwärtsrisiken klar von der Angebotsseite getrieben werden, richten sich die Sorgen in Deutschland mittelfristig primär auf die Nachfrageseite.

Aus keynesianischer Sicht steht dabei die Konjunktur im Fokus und die Sorge, dass der expansive Kurs der Wirtschaftspolitik zu einer Übernachfrage und einen dadurch ausgelösten Aufwärtsdruck auf Löhne und Preise führen könnte. Dieses Risiko ist in den USA als deutlich höher einzuschätzen als im Euroraum und in Deutschland. So hat die US-Wirtschaft im zweiten Quartal 2021 inren Vorkrisentrend bereits wieder erreicht und könnte in den 
Folgequartalen deutlich darüber hinausschießen. Denn die expansiven Fiskalmaßnahmen während der CoronaKrise vor allem in Form von Steuergutschriften haben die Überschussersparnis der US-Haushalte auf einen Rekordwert von über 2 Billionen US-\$ (etwa $10 \%$ am BIP) steigen lassen. Weitere umfangreiche Konjunkturprogramme stehen vor der Verabschiedung durch den Kongress. Auch in Deutschland dürfte die Wirtschaftsleistung 2022 ihr Vorkrisenniveau erreichen und damit früher als im Euroraum. Ein deutliches Überschießen der Nachfrage über das Produktionspotenzial wird, anders als für die USA, aber nicht erwartet, auch da die Fiskalpolitik in den kommenden Jahren deutlich weniger expansiv ausgerichtet sein dürfte (OECD, 2021). Knappheiten am Arbeitsmarkt dürften deshalb auf einzelne Branchen begrenzt bleiben. Ohnehin hat sich der Zusammenhang zwischen Auslastungsgrad am Arbeitsmarkt und Lohnund Preisdynamik in den vergangenen Jahren auch in Europa und Deutschland deutlich verflacht.

Aus monetaristischer Sicht liegt das größte Risiko für die Preisstabilität ohnehin in einer zu expansiven Geldpolitik, die aktuell vor allem an der enormen Steigerung der vom Eurosystem geschaffenen Liquidität festgemacht wird. Deutschland gilt dabei als besonders exponiert. Denn die EZB orientiert sich bei der Kalibrierung ihrer Maßnahmen am Durchschnitt im Euroraum, wodurch die Geldpolitik für das konjunkturell führende Land als notwendigerweise zu expansiv ausgerichtet gilt. So einfach ist es mit dem Inflationsrisiko durch die expansive Geldpolitik aber aus mehreren Aspekten heraus nicht.

Zum einen fußt die Sorge auf der Annahme, dass sich die hohe Notenbankliquidität in einen Anstieg der breiteren Geldmenge überträgt, also den Geldbestand, der zwischen Geschäftsbanken, Unternehmen und Haushalten zirkuliert und so für Preisauftrieb sorgt. Der Zusammenhang zwischen Notenbankliquidität und Geldmenge hat sich in den letzten Jahren aber weiter gelockert. Das liegt auch an der Art der Geldpolitik der EZB. Denn die Banken horten die via Quantitative Easing (QE) in Umlauf gebrachten enormen Liquiditätsüberschüsse beim Eurosystem, aktuell 4,3 Billionen Euro. Dabei kommen die deutschen Banken auf einen Anteil von rund $30 \%$ (Bundesbank, 2021b). Die Mittel haben allerdings kaum Einfluss auf die Geldmenge. Denn in der Praxis wird der Großteil der Geldmenge nicht durch die Liquidität der Notenbank, sondern durch die Geschäftsbanken selbst im Zuge der Kreditgewährung geschaffen (Giralgeld). Zwar hat die EZB mit ihrer expansiven Liquiditätspolitik limitierende Faktoren für die Geldschöpfung der Banken stark aufgeweicht. Dennoch ist die breitere Geldmenge M3 bisher nur moderat gestiegen, und der zeitweise kräftigere Anstieg im Zuge der Pandemie hat sich zu einem Teil bereits wieder zurückgebildet. Denn die Dynamik der Kreditvergabe an die Unternehmen bleibt gering, der Großteil des Anstiegs von M3 geht auf eine verstärkte Kreditaufnahme der öffentlichen Hand zurück.

Zum anderen zeigt die Historie, dass selbst ein deutlicher Anstieg der Geldmenge keinen Schub der Inflation auslösen muss. So liegt das Geldmengenwachstum im Euroraum seit 2000 durchschnittlich deutlich höher als die Inflation. Der Grund lässt sich anhand der Quantitätsgleichung gut zeigen. Denn die Annahme einer konstanten Umlaufgeschwindigkeit ist nicht haltbar. Deshalb mündet selbst ein nachhaltiger Anstieg der zirkulierenden Geldmenge nicht notwendigerweise in ein steigendes Nachfrageverhalten nach Dienstleistungen und Gütern. Bei einem strukturellen Anstieg der Vorsichtskassenhaltung von Haushalten und Unternehmen werden diese das Geld nicht in Umlauf bringen. Im Euroraum und in Deutschland zeigt die Entwicklung der Umlaufgeschwindigkeit genau dieses Verhalten bereits seit 2000. Im Zuge der CoronaKrise hat sich die Entwicklung nochmals verstärkt und die Quotienten aus BIP und M3 sind auf 0,8 gefallen, d.h. jeder Euro der Geldmenge wird durchschnittlich weniger als ein Mal pro Jahr für BIP-relevante Transaktionen eingesetzt. Die Geldschöpfung wirkt weniger aktiv auf die Konjunktur. Der starke Anstieg der Marktkapitalisierung von Aktien und Anleihen ist ein weiteres Indiz für diesen Effekt. Zusammengenommen muss ein Anstieg von Liquidität und Geldmenge also nicht die Verbraucherpreise treiben, sondern kann sein Ventil auch mittelfristig in einer höheren Liquiditäts- und Vorsichtskassenhaltung und/ oder steigenden Vermögenspreisen finden. Dies findet aktuell in einem hohen Maße statt.

\section{Inflationsrisiken im Bereich der strukturellen} Weichenstellungen

Mit Blick auf mögliche Inflationsrisiken durch die Geldpolitik sollte neben der Quantität deshalb auch die Qualität der bereitgestellten Liquidität beachtet werden. So stellen Erklärungsansätze für die Entstehung von historischen Hochinflationsphasen vermehrt auf die Art der Liquiditätsausweitung ab. Demnach ist die Bereitstellung von Notenbankliquidität im Austausch gegen bonitätsstarke Assets (z.B. Ankäufe von Staatsanleihen im QE-Programm) lediglich ein Tausch innerhalb der liquiden Vermögenstitel des Portfolios der Liquiditätsempfänger. Dieser ändert wenig am Verhältnis von liquiden und illiquiden Titeln und hat damit auch nur eine geringe Wirkung auf die Transaktionsmöglichkeiten bzw. die Transaktionsbereitschaft. Zudem wird die Liquidität von den Notenbanken gegen eine Sicherheit (Vermögenstitel) bereitgestellt, die beispielsweise zur Finanzierung von Investitionen und damit Wirtschaftsleistung geschaffen wurde. Solange die 
so geschaffene Wirtschaftsleistung und damit der Vermögenstitel als werthaltig eingestuft werden, stellt der Austausch gegen Liquidiät kein zusätzliches Risiko für die Geldstabilität dar. Deshalb stellen diese Erklärungen für Inflation nicht auf die Liquiditäts- oder Geldmenge per se $a b$, sondern auf die Qualität der zugrunde liegenden Assets, die auf der Aktivseite der Notenbankbilanz ablesbar ist. Demnach steigt durch die expansive Geldpolitik vor allem dann das Inflationsrisiko, wenn die durchschnittliche Werthaltigkeit der Assets schwindet, auch da das Eigenkapital und damit die Qualität der Notenbankbilanz leidet. Die Beurteilung der Asset-Qualität wird dadurch erschwert, dass die Bonitätseinschätzungen und die Marktbepreisung der Assets durch die Effekte der expansiven Geldpolitik beeinflusst werden.

Auch deshalb werden verschiedene Maße vorgeschlagen. Hierzu zählen insbesondere die Anteile von Gold, Devisenreserven und bonitätsstarken Staatsanleihen im Verhältnis zur Geldbasis. Je höher diese Anteile, desto höher wird die Qualität der Liquidität/der Geldbasis eingestuft. Die Bedeutung von Gold wird mit der historischen Stabilität des Goldpreises und der Rolle von Gold als sichere Anlage begründet. Die Bedeutung von (werthaltigen) Devisenreserven fußt vor allem auf der Möglichkeit, den AuBenwert des Geldes durch Devisenmarktinterventionen zu verteidigen und damit einer durch Abwertung ausgelösten Inflationierung entgegenzuwirken. Die Anteile von Gold und Devisenreserven in der Bilanz des Eurosystems sind mit der Ausweitung der Anleihekaufprogramme in einer zweiten Welle ab 2015 weiter gesunken und liegen nur noch bei etwa $15 \%$ der Geldbasis.

Ein ähnliches Bild ergibt sich bei Berücksichtigung von Staatsanleihen und anderen Wertpapieren mit hoher Bonität (AAA). Auch in dieser Betrachtung zeigt sich ein Verlust an Qualität der Geldbasis, der im Fall von noch deutlich steigenden Käufen bonitätsschwächerer Assets an Schärfe gewinnen könnte. Zudem besteht das Risiko, dass sich die Einschätzung der Werthaltigkeit der Anleihen kurzfristig ändert, vor allem wenn Rating-Agenturen ihre Bonitätseinschätzungen anpassen. Die Notenbank hat hierauf nur sehr begrenzt Einfluss. Mit Blick auf die Diskussion der Inflationsrisiken der Geldpolitik sollte die Qualität der angekauften Wertpapiere gerade im Euroraum und in Deutschland deshalb nicht aus dem Blick geraten.

Auch auf der Angebotsseite liegen Risiken für die Preisstabilität, die nicht mit dem engen Blick auf die Wirtschaftspolitik abgedeckt sind. Die politisch angestrebte Internalisierung der Kosten von $\mathrm{CO}_{2}$-Emissionen und weiteren Aspekten des Klimawandels könnte die Produktionskosten über höhere Energiepreise mittelfristig erheblich steigern. Preisanstiege in diesen Bereichen sind politisch gewollt.
Gleichzeitig dominiert die Einschätzung, wonach in anderen Bereichen mit Kosten- und Preissenkungen zu rechnen ist. So sollen klimafreundliche Geschäftsmodelle etwa von günstigeren Finanzierungskonditionen profitieren und diese Vorteile an die Verbraucher:innen weitergeben. Gesamtwirtschaftlich dürfte die angestrebte Berücksichtigung von externen Effekten aber in den kommenden Jahren preissteigernd wirken, auch da die Produktivitätsfortschritte begrenzt bleiben dürften.

Gleiches gilt für Maßnahmen zur Re-Nationalisierung von Produktion in Reaktion auf die Corona-Krise und die globalen Handelsstreitigkeiten. Eine höhere Resilienz der Wertschöpfungsketten auch über höhere Lagerhalterungen wird nicht kostenlos zu haben sein. Dadurch könnte sich auch der disinflationäre Trend von Produktionsverlagerungen in Niedriglohnländer der vergangenen Jahre abschwächen bzw. sogar umkehren. In die gleiche Richtung wirken die international diskutierten Effekte einer zunehmend alternden Bevölkerung und dem damit verbundenen Rückgang des Angebots an Arbeitskräften in vielen Ländern, die nach Deutschland exportieren und/ oder als Produktionsstandorte genutzt werden, vor allem China. Diese Faktoren sollten mit Blick auf die Debatte zum Inflationsausblick in Deutschland und dem Euroraum ein stärkeres Gewicht erhalten.

Und schließlich stellt sich auch die Frage, ob die zu beobachtenden Anstiege von Vermögenspreisen nicht stärker in der Inflationsberechnung berücksichtigt werden sollten. Dies gilt insbesondere für den Immobilienmarkt. Denn die Wohnkosten stehen für einen erheblichen und weiter steigenden Teil der Lebenshaltungskosten auch in Deutschland und sollten nicht nur als Assetpreise klassifiziert werden. Hier hat die EZB im Rahmen ihres Strategieprozesses eine Anpassung vorgeschlagen, welche die Inflationsdebatte in den kommenden Jahren erneut entfachen könnte.

\section{Inflationsdebatte weniger dogmatisch führen, Blick auf Megatrends richten}

Weder die Liquiditätsschwemme durch die EZB noch der expansive Kurs der Fiskalpolitik macht eine höhere Inflation unausweichlich. Eine aufgeregte Diskussion und ein zu enger Blick sind auch deshalb problematisch, da strukturellen Faktoren dadurch zu wenig Beachtung geschenkt wird. Hier könnten die Inflationspotenziale und -risiken mittelfristig deutlich größer sein. Auch deshalb sollte eine Diskussion über Inflationsrisiken in Deutschland die strukturellen Weichenstellungen und Entwicklungen stärker in den Blick nehmen. Denn hier stehen sich preistreibende und preissenkende Faktoren unmittelbar gegenüber und müssen gegeneinander abgewogen werden. 


\section{Literatur}

Bundesbank (2021a), Inflationsraten, die in Richtung 5 Prozent gehen, https://www.bundesbank.de/de/presse/interviews/-inflationsraten-diein-richtung-5-prozent-gehen--869908 (24. Juli 2021).

Bundesbank (2021b), Bundesbank Monatsbericht Januar 2021, https://www.bundesbank.de/resource/blob/856554/504763e0dcde
7f55e3f054873f6af199/mL/2021-01-tiering-data.pdf (30. August 2021).

Destatis (2021), Inflationsrate im Juli 2021 bei $+3,8 \%$, https://www.destatis.de/DE/Presse/Pressemitteilungen/2021/08/PD21_377_611.html (30. August 2021).

OECD (2021), OECD Economic Outlook, Volume 2021, Issue 1, https:// www.oecd.org/economic-outlook/ (30. August 2021). 